\title{
A Lower Bound on the Angles of Triangles Constructed by Bisecting the Longest Side
}

\author{
By Ivo G. Rosenberg and Frank Stenger
}

\begin{abstract}
Let $\triangle A^{1} A^{2} A^{3}$ be a triangle with vertices at $A^{1}, A^{2}$ and $A^{3}$. The process of "bisecting $\triangle A^{1} A^{2} A^{3}$ " is defined as follows. We first locate the longest edge, $A^{i} A^{i+1}$ of $\Delta A^{1} A^{2} A^{3}$ where $A^{i+3}=A^{i}$, set $D=\left(A^{i}+A^{i+1}\right) / 2$, and then define two new triangles, $\Delta A^{i} D A^{i+2}$ and $\Delta D A^{i+1} A^{i+2}$.

Let $\Delta_{00}$ be a given triangle, with smallest interior angle $\alpha>0$. Bisect $\Delta_{00}$ into two new triangles, $\Delta_{1}, i=1,2$. Next, bisect each triangle $\Delta_{1 i}$, to form four new triangles $\Delta_{2 i}, i=1,2,3,4$, and so on, to form an infinite sequence $T$ of triangles. It is shown that if $\Delta \in T$, and $\theta$ is any interior angle of $\Delta$, then $\theta \geqslant \alpha / 2$.
\end{abstract}

Results. Let $\triangle A B C$ be a triangle with vertices at $A, B$ and $C$. The procedure "bisect $\triangle A B C$ " is defined as follows. We form two triangles from $\triangle A B C$ by locating the midpoint of the longest side of $\triangle A B C$ and drawing a straight line segment from this midpoint to the vertex of $\triangle A B C$ which is opposite the longest side. (If there is more than one side of greatest length, we bisect any one of them.) For example, if $B C$ is the longest side of $\triangle A B C$, we set $D=(B+C) / 2$ to form two new triangles $\triangle A B D$ and $\triangle A D C$.

Let $\triangle A B C$ be a given triangle with interior angles $\alpha, \beta$ and $\gamma$ located at $A, B$ and $C$, respectively. We form an infinite family $T(A, B, C)$ of triangles as follows. We first bisect $\Delta_{00} \equiv \triangle A B C$ to form two new triangles $\Delta_{1}, i=1,2$. We next bisect each of these two triangles to form four new triangles $\Delta_{2 i}, i=1,2,3,4$. Next, we bisect each of these four triangles to form eight new triangles $\Delta_{3 i}, i=1,2,3, \ldots$, 8 , and so on.

It is convenient to apply this procedure of bisections in order to refine the mesh in the finite element approximations of solutions of differential equations (see, e.g., [1]). Recently [2], this procedure of bisecting triangles was used to obtain a two-dimensional analogue of the one-dimensional method of bisections for solving nonlinear equations. A criterion of convergence of the above procedures is that the interior angles of $\Delta_{n i}$ do not go to zero as $n \rightarrow \infty$. The Schwarz paradox [3,pp.373-374] provides an explicit example of a situation in which triangles are used to approximate the area of a cylinder. In this case, the sum of the areas may not converge to the area of the cylinder as the length of each side of the triangles approaches zero, and the number of triangles approaches infinity, if the smallest interior angle of each triangle approaches zero.

Received January 2, 1974.

AMS (MOS) subject classifications (1970). Primary 50B30, 50B15; Secondary $41 \mathrm{A63}$, $65 \mathrm{~N} 30,65 \mathrm{H} 10$.

Key words and phrases. Triangulation, bisection, finite element method. 
In this note, we prove the following theorem, which ensures that the interior angles of $\Delta_{n i}$ do not go to zero as $n \rightarrow \infty$.

THEOREM. Let the smallest interior angle of $\triangle A B C$ be $\lambda$, and let $0<x_{\lambda}<\pi / 4$ be the solution of

$$
\tan x_{\lambda}=\frac{\sin \lambda}{2-\cos \lambda}
$$

If $\Delta$ is a triangle in $T(A, B, C)$, and $\theta$ is an interior angle of $\Delta$, then $\theta \geqslant x_{\lambda}$.

CoRollary. If $\Delta \in T(A, B, C)$ and $\theta$ is an interior angle of $\Delta$, then $\theta \geqslant \lambda / 2$.

In the case when $\lambda$ is small, $x_{\lambda}$ is a better lower bound than $\lambda / 2$, since $x_{\lambda} / \lambda \rightarrow$ 1 as $\lambda \rightarrow 0$. For example, when $\lambda=\pi / 6, x_{\lambda} \cong .777(\pi / 6)>.5(\pi / 6)=\lambda / 2$.

Before we start the proof of the above theorem and corollary, we introduce the following notation.

Let $\triangle R S T$ be a triangle with interior angles $\rho, \sigma$ and $\tau$ at $R, S$ and $T$, respectively. If $\triangle R S T$ is bisected into two triangles $\Delta R_{i} S_{i} T_{i}$ with interior angles $\rho_{i}, \sigma_{i}$ and $\tau_{i}$ located at $R_{i}$, $S_{i}$ and $T_{i}$, respectively, $i=1,2$, we use both the notations

$$
(\rho, \sigma, \tau) \longrightarrow\left(\rho_{i}, \sigma_{i}, \tau_{i}\right), \quad\left(\rho_{i}, \sigma_{i}, \tau_{i}\right) \longleftarrow(\rho, \sigma, \tau)
$$

As the notation suggests, $(\rho, \sigma, \tau)$ actually denotes a similarity class in $T(A, B, C)$ and " $\rightarrow$ " is a binary relation, or graph, on the set of all these similarity classes. We also use the notation $|M-N|$ to denote the Euclidean distance between the points $M$ and $N$.

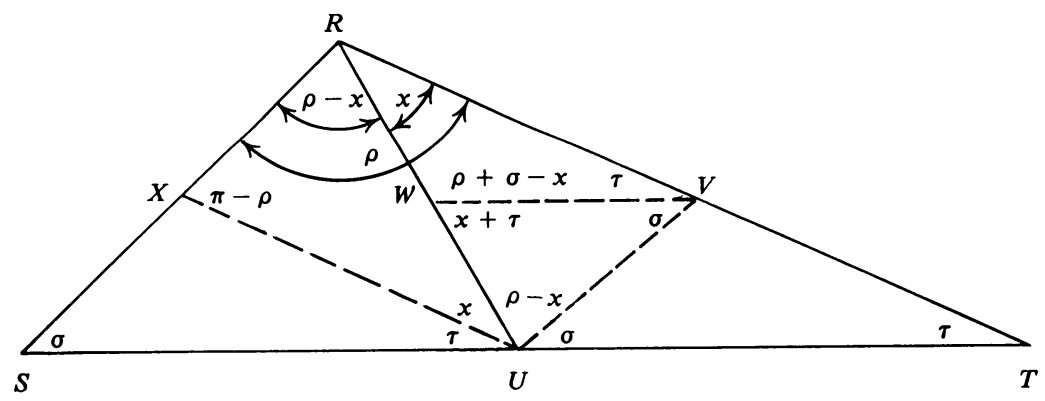

FIGURE 1. Bisections of a triangle $\triangle R S T$

Proof of the Theorem. Let $\triangle R S T$ (see Fig. 1) belong to the family $\Delta(A, B, C)$, and let $\Delta R S T$ have interior angles $\rho$ at $R, \sigma$ at $S$, and $\tau$ at $T$. Let us also assume without loss of generality that $0<\tau \leqslant \sigma \leqslant \rho$. Since also $\rho+\sigma+\tau=\pi$, it follows that

$$
\tau \leqslant \pi / 3 \leqslant \rho<\pi . \quad \text { and } \quad \sigma<\pi / 2
$$


From Fig. 1, we obtain

$$
(x, \tau, \rho+\sigma-x) \leftarrow(\rho, \sigma, \tau) \rightarrow(\rho-x, \sigma, x+\tau) .
$$

Since the sizes of the sides of $\Delta R S T$ are in the same relation as the opposite angles, from $\sigma \geqslant \tau$ we first get $|T-R| \geqslant|S-R|$, and then, applying the same principle to $\Delta R V U$, the relations $|V-R|=1 / 2|T-R| \geqslant 1 / 2|S-R|=|V-U|$ yield

$$
x \leqslant \rho-x .
$$

Lemma 1. Let $\tau \leqslant \pi / 3$ and $\rho=\sigma=\pi / 2-\tau / 2$. Then the angle $x_{\tau}$ in Fig. 2 satisfies

$$
\tan x_{\tau}=\frac{\sin \tau}{2-\cos \tau} \geqslant \tan \tau / 2
$$

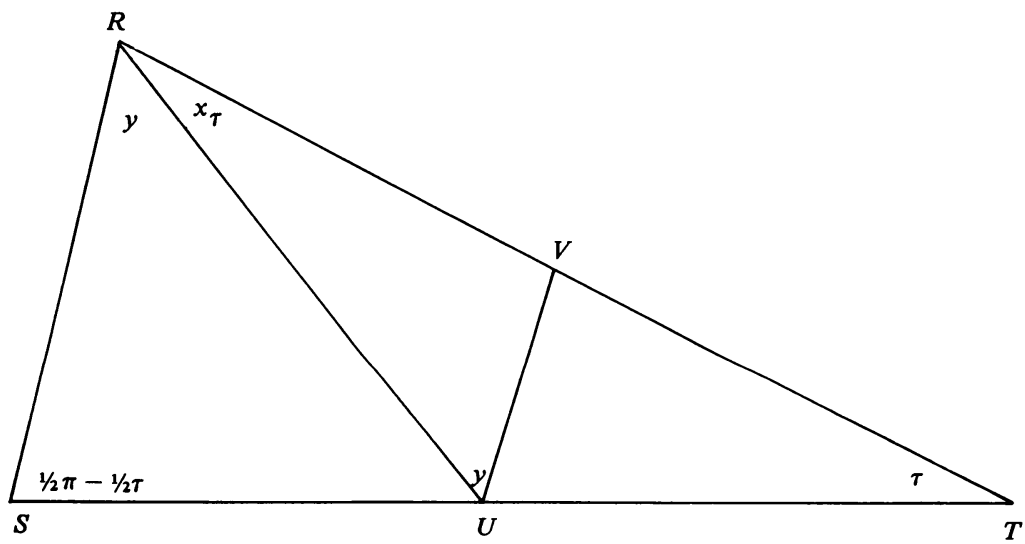

FIGURE 2. $\Delta R S T$ when $|R-T|=|S-T|$

Proof. The law of sines in $\triangle R U V$ and $\triangle R S T$ yields

$$
\frac{\sin x_{\tau}}{\sin y}=\frac{|V-U|}{|V-R|}=\frac{|S-R|}{|T-R|}=\frac{\sin \tau}{\sin (\pi / 2-\tau / 2)} .
$$

Since $y=\pi / 2-\tau / 2-x_{\tau}$, we obtain

$$
\sin x_{\tau} \cos 1 / 2 \tau=\sin \tau \cos \left(\tau / 2+x_{\tau}\right) .
$$

Simplifying, we get $\tan x_{\tau}=\sin \tau /(2-\cos \tau)$. From the relation $2 z \equiv \tau \leqslant \pi / 3$, we get $\cos ^{2} z-\sin ^{2} z=\cos 2 z \geqslant 1 / 2$, and hence $2 \cos ^{2} z \geqslant 1+2 \sin ^{2} z=2-\cos 2 z$. This yields $2 \sin z \cos z /(2-\cos 2 z) \geqslant \tan z$, which proves Lemma 1 . 


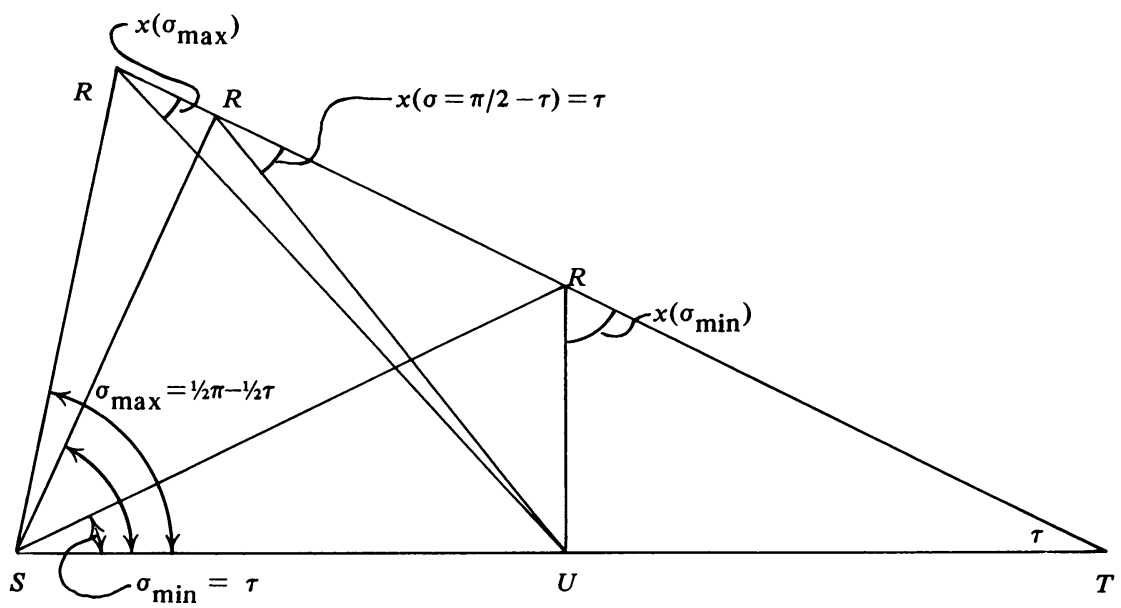

FIGURE 3. Various values of the angle $x_{\tau}$

With reference to Fig. 3, let us fix $S, T$, and the angle $\tau$, and change $\sigma$ so that $\rho \geqslant \sigma \geqslant \tau$. Clearly, $\sigma$ changes from $\sigma_{\min }=\tau$ to $\sigma_{\max }=\pi / 2-\tau / 2$, when $\Delta R S T$ becomes an isosceles triangle. Clearly, $x=x(\sigma)$ is a decreasing function of $\sigma$ in the region $\sigma_{\min } \leqslant \sigma \leqslant \sigma_{\max }$, whose range of values are

$$
x_{\tau}=x\left(\sigma_{\max }\right) \leqslant x(\sigma) \leqslant x\left(\sigma_{\min }\right)=\pi / 2-\tau,
$$

where $x_{\tau}$ is defined in (6). Thus by Lemma 1 ,

$$
x \geqslant x_{\tau} \geqslant \tau / 2
$$

Notice also that when $\rho=\pi / 2, \sigma=\pi / 2-\tau$, and $x(\sigma)=x(\pi / 2-\tau)=\tau$. It is thus evident from Fig. 3, that

$$
x \geqslant \tau \Longleftrightarrow \rho \geqslant \pi / 2
$$

Finally, we remark that $x_{\tau}$ is an increasing function of $\tau$ in the region $0 \leqslant \tau \leqslant \pi / 3$, which can be easily verified by computing the derivative of $x_{\tau}$ using (6).

We next show that

$$
x+\tau \leqslant \pi / 2, \quad \rho+\sigma-x \geqslant \pi / 2
$$

For if $x+\tau>\pi / 2$, then, since the interior angles of $\Delta R S U$ in Fig. 1 add up to $\pi$, it would follow that $\rho+\sigma-x<\pi / 2$. However, from (5), we get $\rho-x \geqslant \rho / 2$, and so $\sigma+\rho / 2<\pi / 2$, i.e., $\rho+2 \sigma<\pi$. Since, however, $\rho+2 \sigma \geqslant \rho+\sigma+\tau=\pi$, we arrive at a contradiction, i.e., (9) is valid.

In view of (9), we establish 
LEMma 2. The following situation

(10)

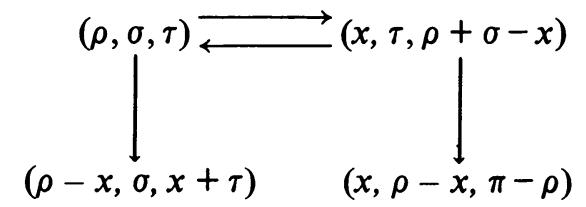

is valid in general.

LEMMA 3. If

$$
\pi-\rho \geqslant \rho-x,
$$

then

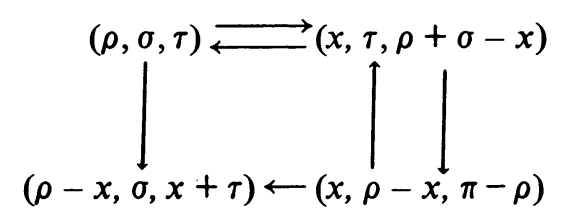

Proof of Lemma 3. By combining (11) and (5), it follows that $\pi-\rho \geqslant \rho-x$ $\geqslant x$, and (12) now follows by inspection of $\triangle R X U$ or $\triangle R U V$.

We next consider the bisection of $\triangle W U V$ or $\triangle R S U$.

Lemma 4. Let (11) hold. If

$$
x+\tau \geqslant \sigma \text { and } x+\tau \geqslant \rho-x
$$

or else if

$$
\rho-x<\tau
$$

then

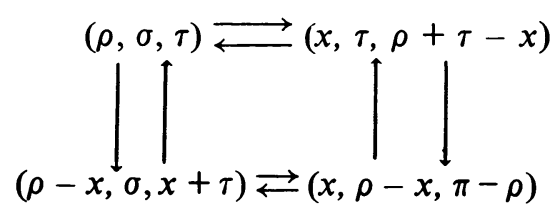

Proof. If (13) is satisfied, then (15) clearly follows from (12) and inspection of $\Delta W U V$ in Fig. 1. If (14) holds, then $\rho-x<\tau<x+\tau$, so that the second relation in (13) is satisfied. If the first relation of (13) were not satisfied, then $\sigma>x+\tau$, and, by (14), $x+\tau>x+\rho-x=\rho$, i.e., $\sigma>\rho$, which contradicts our original assumption, that $\tau \leqslant \sigma \leqslant \rho$. This proves Lemma 4 .

Let us now complete the proof of the theorem. Let us set $\nu=\nu(\rho, \sigma, \tau)=$ $\min (\rho, \sigma, \tau)$. We shall show that, along the transition $\rightarrow$, either (i) $\nu$ is nondecreasing, or (ii) we get four triples $t_{i}=\left(\rho_{i}, \sigma_{i}, \tau_{i}\right)$ such that $\nu=\nu\left(t_{i}\right) \geqslant x_{\tau}, \quad i=1,2,3,4$, and such that if an arrow emanates from one of the four triples, $t_{j}$, to a triple $t$ where 
$t \neq t_{i}, \quad i=1,2,3,4$, then $v\left(t_{j}\right) \geqslant \tau$. Since $x_{\tau}$ is an increasing function of $\tau$, it will therefore be impossible to get $\nu\left(\Delta_{n i}\right)<x_{\lambda}$ for any $\Delta_{n i} \in T(A, B, C)$.

Let us assume that $\triangle R S T$ is an arbitrary triangle in the family $T(A, B, C)$, such that $\rho \geqslant \sigma \geqslant \tau$. In (10), $\rho+\sigma-x \geqslant \pi / 2>\tau, \sigma \geqslant \tau, x+\tau \geqslant \tau$, and $\pi-\rho=\sigma+$ $\tau \geqslant \tau$. Thus, the only candidates for angles $<\tau$ are $x$ and $\rho-x$. If $\rho \geqslant \pi / 2$, it follows from (5) and (8) that $\rho-x \geqslant x \geqslant \tau$, and hence $\nu(\rho-x, \sigma, x+\tau) \geqslant \nu(x, \tau, \rho+\sigma-x)$ $\geqslant \nu(\rho, \sigma, \tau)$.

Let us assume, therefore, that $\rho<\pi / 2$. Then $\pi-\rho>\pi / 2>\rho>\rho-x$, so that (11) is satisfied, and, by Lemma 3 , we get the situation (12). In (12), the inequalities $\sigma \geqslant \tau, \rho+\sigma-x \geqslant \pi / 2$, and $\pi-\rho \geqslant \pi / 2$ are valid, and so only the angles $x$ and $\rho-x$ can be less than $\tau$. By (7) and (5) $\rho-x \geqslant x \geqslant x_{\tau}$. The configuration (12) is such that arrows going outside of it can originate only at $(\rho-x, \sigma, x+\tau)$. If $\rho-x \geqslant \tau$, then $\nu(\rho-x, \sigma, x+\tau) \geqslant \tau$.

Finally, if $\rho-x<\tau$, we have the situation of Lemma 4 , that is the four triples $\left(\rho_{i}, \sigma_{i}, \tau_{i}\right)$ form a "trap" in the sense that there are no arrows emanating from them, and such that $\nu\left(\rho_{i}, \sigma_{i}, \tau_{i}\right) \geqslant x_{\tau}, i=1,2,3,4$.

This clearly completes the proof.

Centre de Recherches Mathématiques

Université de Montréal

Montréal, Québec, Canada

Department of Mathematics

University of Utah

Salt Lake City, Utah 84112

1. M. ZLÁMAL, "On some finite element procedures for solving second order boundary value problems," Numer. Math., v. 14, 1969/70, pp. 42-48. MR 41 \#1244.

2. C. HARVEY \& F. STENGER, "A two dimensional extension of the method of bisections for solving nonlinear equations," Quart. Appl. Math. (to appear.)

3. J. F. RANDOLPH, Calculus and Analytic Geometry, Wadsworth, Belmont, Calif., 1961. 